\title{
VITAMIN A AND CAROTENE. II. VITAMIN A AND CAROTENE METABOLISM IN DIABETICS AND NORMALS
}

\author{
By WILLIAM A. MURRILL, PRISCILLA B. HORTON, ESTHER LEIBERMAN, \\ AND L. H. NEWBURGH
}

(From the Department of Internal Medicine, University of Michigan, Ann Arbor) ${ }^{1}$

(Received for publication February 26, 1941)

Investigation of vitamin $\mathrm{A}$ and carotene metabolism in diabetes mellitus has led to several interesting observations by different workers. Ralli, et al (1) have shown that the blood level of carotene is higher in the diabetic than in the normal. She also found that the administration of carotene in oil to diabetic patients caused the blood carotene to rise to a higher level and to remain there longer than in the normal controls. From this Ralli postulated that in diabetes there is a diminished ability of the liver to convert carotene to vitamin A. Brazer and Curtis (2) found supporting evidence for this theory in work based on the Jeans biophotometer test. They reported that dark adaptation was abnormally slow in patients with diabetes mellitus, indicating vitamin A deficiency. The administration of carotene to these patients caused no change in the biophotometer reading, whereas vitamin $A$, in equivalent amounts, quickly returned their dark adaptation time to normal.

Recent work in our laboratory (3), using rats, has shown that the concentration of vitamin $A$ in the blood is a reliable index to the vitamin A status of the body. In view of this, we felt that it was of interest to investigate further the vita$\min \mathrm{A}$ of the diabetic individual and to examine the theory postulated by Ralli. This was done by following the blood vitamin A and carotene levels of normals and of patients with diabetes mellitus.

\section{EXPERIMENTAL AND DISCUSSION}

Vitamin A and carotene were determined by Kimble's (4) modification of the method of Dann and Evelyn (5), using the macro unit of the Evelyn photoelectric colorimeter. Vitamin $A$ is expressed as micrograms per 100 cc. of plasma. This is derived from the fundamental $L$ ( 1 per cent, $1 \mathrm{~cm} ., 620 \mathrm{~m} \mu$ ) values, calculated according to Kimble (4). By converting $L$ (1 per cent, $1 \mathrm{~cm}$.,

1 The expense of this study was defrayed in part by a grant from the Horace $H$. Rackham and Mary A. Rackham Foundation.
$620 \mathrm{~m} \mu$ ) to the spectroscopic unit $E$ (1 per cent, $1 \mathrm{~cm}$., $328 \mathrm{~m} \mu$ ) (5), and applying the value for crystalline vitamin A determined by Holmes and Corbet (6), it is possible to express our results as micrograms per 100 cc. Carotene, which is also recorded in micrograms per 100 cc., was calculated from a standard reference curve, using two sources of crystalline carotene (Eastman Kodak Company 3702 and Nutritional Research Associates, Inc.) with color filter 440. By this method, plasma vitamin $\mathrm{A}$ and carotene concentrations were determined on a number of healthy controls (physicians, dietitians, nurses, and laboratory workers, all eating in the same dining room). The blood on which the

TABLE I

Carotene and vitamin $A$ blood values of normal subjects

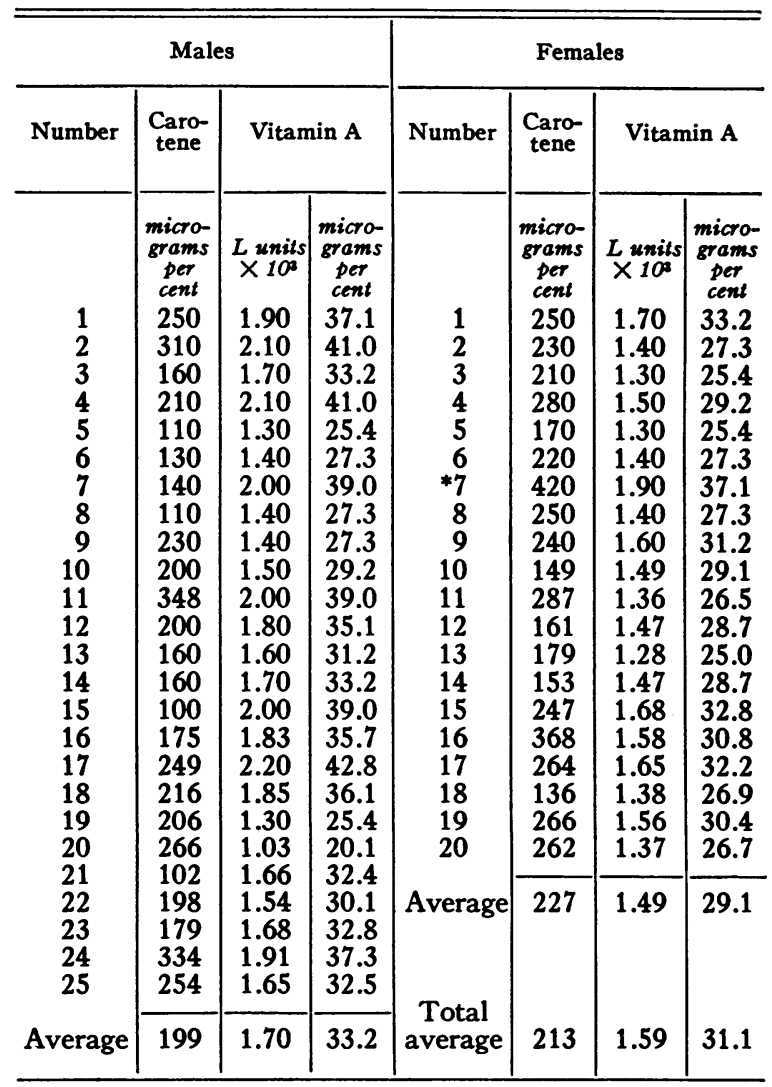

* One healthy dietitian on approximately the same diet as the others had a consistently higher plasma carotene. Because of the great difference, we felt justified in omitting it from the normal series. 
determinations were made was not necessarily fasting blood, since Kimble (4) has shown that ordinary meals do not increase the concentration of either vitamin A or carotene within two to six hours after ingestion. The range was found to be 20.0 to 43.0 micrograms of vitamin A per $100 \mathrm{cc}$. and 100 to 368 micrograms of carotene per $100 \mathrm{cc}$, as shown in Table I. The vitamin A concentrations in this group were close to, though somewhat higher, than those obtained in rats that were shown to have adequate stores in their liver. This seems to indicate that the normal subjects used were all in a good vitamin $\mathrm{A}$ nutritive state. In addition, our range is in close agreement with that found by Kimble (4) (1.2 to $2.9 \mathrm{~L} 620$ units of vitamin $\mathrm{A}$ and 100 to 360 micrograms of carotene per $100 \mathrm{cc}$.) on a larger group of healthy controls. Our data show a sex variation, which, again, is in agreement with Kimble. The women have slightly lower vitamin A and higher carotene values than the men. The difference, however, is of doubtful significance.

Results on sixteen diabetic patients are shown in Table II. Those included in the study were chosen because they appeared to be of the juvenile type and were selected from the patients who presented themselves to the clinic for care. Except for the three from whom we obtained carotene tolerance data, we collected our samples without interfering with the clinical treatment and in only a few cases were the patients glycosuric. During the experimental period, the three patients on whom carotene tolerance tests were made were

TABLE II

Carotene and vitamin $A$ blood values for diabetic subjects

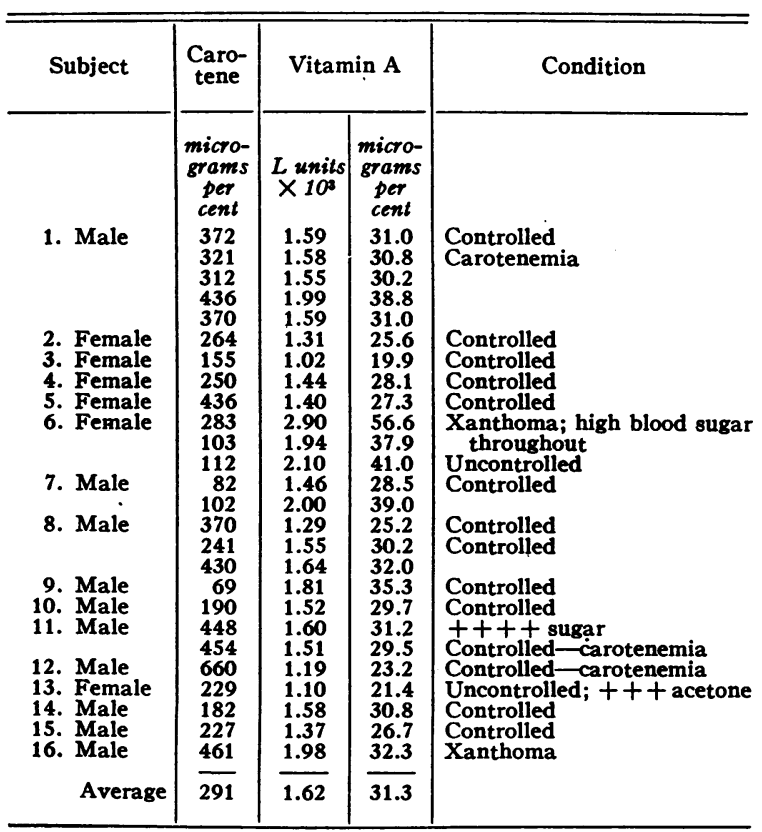

controlled with insulin. Two of them remained aglycosuric throughout the period and the third showed a small amount of sugar in two of four daily urine specimens. The ages of all patients ranged from 13 to 41 years. Ten were under 25, the remaining six over 25 . All presented a history of the typical onset of the disease-polyuria, polyphagia, polydipsia and weight loss. Twelve had ketonuria on various admissions and of the remaining four, three were admitted in diabetic coma and the fourth gave a history of such an attack. In every case the fasting blood sugar before treatment was more than 187 milligrams per cent. With the use of diets containing from 150 to 300 grams of carbohydrate, it was necessary to use insulin in every case in amounts ranging from 30 to 105 units daily. The diabetes in these sixteen patients ranged from moderate to greatest severity, as would be expected in any such group of young diabetics.

The vitamin A values for the juvenile diabetics are surprisingly constant. With the exception of one high value ( $56.6 \mathrm{mgm}$. per $100 \mathrm{cc}$.) in a patient with xanthoma diabeticorum, all fell within the normal range and the average is the same as it is for the normal subjects. The constancy of the vitamin A concentrations in these patients was not related to the widely varying carotene concentrations. A large number of the latter fall above the normal range, and the average is significantly higher than is the carotene average of the normals.

Carotene tolerance data with both normal and diabetic subjects were also obtained. In this way we were able to compare the concentrations of vitamin $A$ and carotene attained in the blood and the subsequent rate of decline in the two classes of subjects after administration of a massive dose of carotene. In all cases several determinations were run during the week prior to administration of carotene to determine the average concentration in the blood for each subject. During this time the subjects were on a standard diet containing 7500 International Units of vitamin A. Eighty milligrams $(130,000$ International Units) of carotene in oil were given by mouth. Blood analyses were made at two, four, six, and nine hours after the administration, and daily thereafter for the ensuing five days. A summary of the results is presented in Figure 1. 


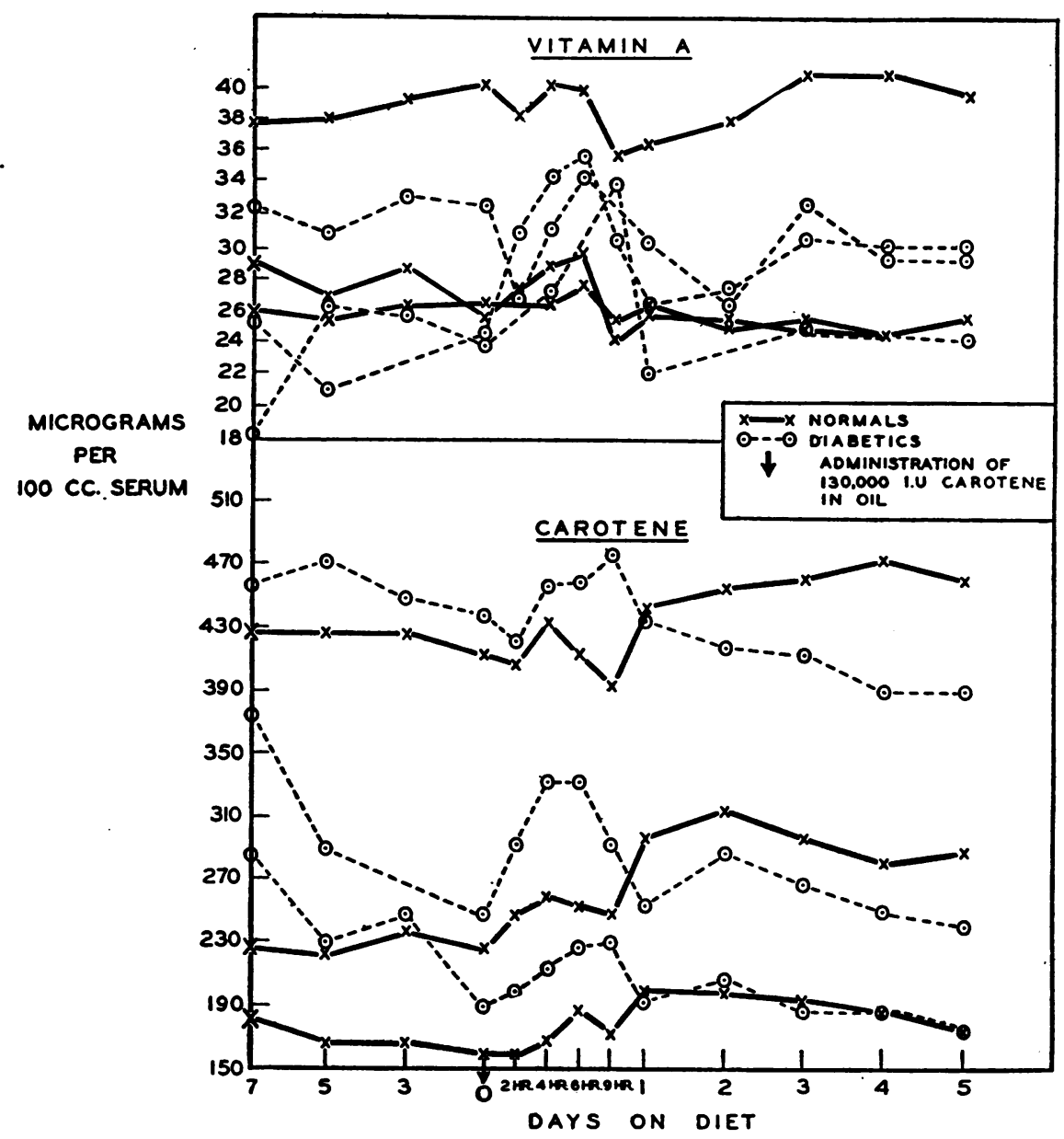

Fig. 1. Blood Levels for Vitamin A and Carotene Before and After Administration of 130,000 I.U. of Carotene in Oil to Diabetic and Normal Subjects

The concentration of carotene in the blood did not rise to as high a level as might be expected, in view of the massive dose given, in either the diabetic or the normal subjects. The stools were observed to be reddish in color, indicating that the carotene was not well absorbed from the intestine. However, the diabetics did not show a greater rise in the concentration of carotene in the blood, nor did the concentrations remain elevated longer than in the normals. The variations in the concentration of vitamin A in the blood did not alter significantly in either group of subjects throughout the experiment. This observation, plus the fact that the entire group of diabetics studied had normal concentrations of vitamin $\mathrm{A}$ in the blood, did not indicate that the liver of the diabetic converts carotene to vitamin A more slowly than the normal. This study, of course, represents a small group of diabetic subjects, and the results are not statistically conclusive. Josephs (8) found that there is a relationship between vitamin $\mathrm{A}$ and carotene on the one hand, and total blood lipids on the other, due to the fat solubility of the former. If this is the case, the high blood fat often associated with diabetes would help to explain the high carotene values found in our diabetic group. This is an aspect of the subject that we are now investigating.

Since the variations in blood vitamin A were so small in our diabetics, we were led to the investigation of vitamin A and carotene in two normal subjects (M. M. and A. C.) placed on vitamin A low diets. In this way we wished to find out how easily and to what extent the carotene and 


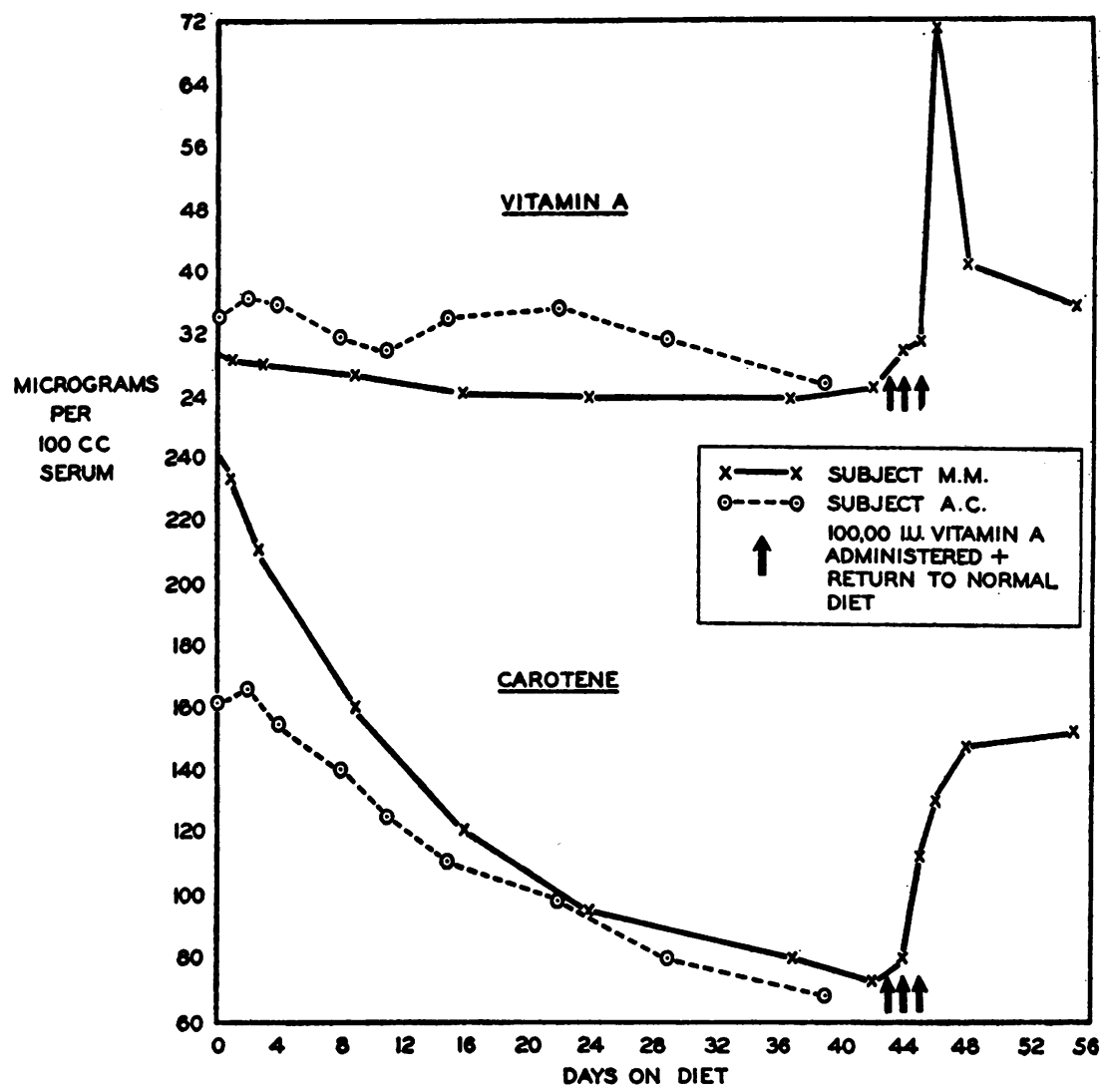

Fig. 2. Blood Vitamin A and Carotene Depletion Values on Two Normal Subjects on a Vitamin A Low Diet

vitamin A values could be lowered and whether there were concomitant physiological symptoms. The diet given was a standard vitamin A-deficient diet and was calculated to furnish 296 International Units of vitamin A per day. M. M. and A. C. remained on this diet for forty-two days and thirty-nine days, respectively. M. M. then returned to her regular diet during the period of administration of vitamin A concentrates. During this time plasma analyses for carotene and vitamin $\mathrm{A}$ were made at regular intervals. These values are given in Figure 2.

It is interesting to note the steady decline in carotene level until definitely subnormal concentrations were reached in both cases. The vitamin $\mathrm{A}$, on the other hand, was lowered only a small amount and, at the end of the depletion period, was still well within the normal range. In the case of M. M., the vitamin A supplement (100,000 International Units as cod liver oil for four days) caused an immediate elevation in vitamin
A, reaching a super-normal concentration (70.5 mgm. per $100 \mathrm{cc}$.) on the third day, then quickly falling to within the normal range. Due to the return to a normal diet, carotene was restored to a normal value in two days. A falling carotene blood level appears to be the first sign of an impending vitamin A deficiency. Since food is the principle source of carotene for the blood, whereas the vitamin A concentrations may be maintained either from the diet or the liver stores, this would be expected. In contrast to Steininger et al (12), who found that the concentration of vitamin $A$ in the blood fell markedly even during the first week on a depletion diet, in the present investigation the concentration was not lowered significantly by forty days on the special diet If the values obtained for vitamin $\mathrm{A}$ in the blood are interpreted in the light of our rat studies (3), they suggest that the liver still had a plentiful store of vitamin A. Even if a drop in the blood level had indicated serious impairment to the liver stores, con- 
siderable time might elapse before physiological symptoms associated with avitaminosis A would assert themselves.

Biophotometric readings according to the Jeans (7) technique were carried out during the course of depletion of M. M. and A. C., and also on various of our diabetic subjects. These data are not included in this paper because results were so variable that it was impossible to draw any conclusions from them. In addition, the reliability of biophotometric data has been widely criticized (9, 10, 11). Hecht and Mandelbaum (10), using a different technique, by which they measured both rod and cone adaptation, state that measurements of dark adaptation, when made under critically standardized conditions, may be used as an aid in the diagnosis of avitaminosis A. Steininger and Roberts $(11,12)$, using the Jeans technique, have concluded that, although there is a relationship between vitamin A deficiency and biophotometer readings, it is not close enough to detect subclinical deficiencies. In addition, they were unable to find any correlation between the biophotometer readings and the concentrations of vitamin $A$ in the blood. Since it is still a controversial subject, we felt that our data would have little significance.

It seems evident that the vitamin A plasma concentration is altered only in extreme cases of vitamin deficiency. The depletion period for the two subjects just discussed was not long enough to reach this critically low level of vitamin A. The diabetic group showed that the disease did not at all affect normal vitamin A concentrations in the blood. However, there is some disturbance in carotene metabolism in diabetes, since many of our patients had clinical carotenemia, as evidenced by yellow pigment deposits, especially in the palms of the hands. Our evidence leads us to conclude that it is not necessarily an impairment of the mechanism that converts carotene to vitamin A.

\section{SUM MARY}

1. The normal blood plasma concentrations for carotene and vitamin $\mathrm{A}$, determined according to the method of Kimble, were found to be 100 to 368 micrograms per 100 .cc. for carotene and 20.0 to 43.0 micrograms per $100 \mathrm{cc}$. for vitamin A.

2. Blood plasma concentrations were determined in patients with diabetes mellitus. The average carotene concentration for the diabetic group was significantly higher than for the normal group, while the vitamin A concentrations were the same.

3. Carotene tolerance curves were run on three normal and on three diabetic subjects. There was no delay in the fall of the carotene concentrations attained in the diabetic subjects after the administration of 80 milligrams $(130,000$ International Units) of carotene. This may be an indication that the carotenemia observed in diabetics is not due to the failure of conversion of carotene to vitamin A.

4. Two normal subjects were depleted for forty-two and thirty-nine days. One was given vitamin A concentrate. During this time records were kept of the blood plasma concentration for carotene and vitamin $\mathrm{A}$. The carotene fell to subnormal levels but the vitamin A was not appreciably changed.

The authors wish to thank Doctor Arthur C. Curtis for his many helpful suggestions and criticisms throughout this study.

\section{BIBLIOGRAPHY}

1. Ralli, E. P., and others, Effect of carotene and vitamin A on patients with diabetes mellitus: effect of daily administration of carotene on the blood carotene of normal and diabetic individuals. J. A. M. A., 1936, 106, 1975.

2. Brazer, J. G., and Curtis, A. C., Vitamin A deficiency in diabetes mellitus. Arch. Int. Med., 1940, 65, 90.

3. Horton, P. B., Murrill, W. A., and Curtis, A. C., The determination of vitamin $A$ in the blood and liver as an index of vitamin A nutrition of the rat. (To be published.)

4. Kimble, M. S., The photocolorimetric determination of vitamin $\mathrm{A}$ and carotene in human plasma. J. Lab. and Clin. Med., 1939, 24, 1055.

5. Dann, W. J., and Evelyn, K. A., The determination of vitamin $A$ with the photoelectric colorimeter. Biochem. J., 1938, 32, 1008.

6. Holmes, H. N., and Corbet, R. E., The isolation of crystalline vitamin A. J. Am. Chem. Soc., 1937, 59, 2042.

7. Jeans, P. C., Blanchard, E., and Zentmire, Z., Dark adaptation and vitamin $A$; a new photometric technic. J. A. M. A., 1937, 108, 451.

8. Josephs, H. W., Relation of vitamin A and carotene to serum lipids. Bull. Johns Hopkins Hosp., 1939, 65, 112. 
9. Isaacs, B. L., Jung, F. T., and Ivy, A. C., Vitamin A deficiency and dark adaptation. J. A. M. A., 1938, 111, 777.

10. Hecht, S., and Mandelbaum, J., The relation between vitamin A and dark adaptation. J. A. M. A., 1939, $112,1910$.

11. Steininger, G., and Roberts, L. J., Biophotometer test as index of nutritional status for vitamin A. Arch. Int. Med., 1939, 64, 1170.

12. Steininger, G., Roberts, L. J., and Brenner, S., Vita$\min \mathrm{A}$ in the blood of normal adults: The effect of a depletion diet on blood values and biophotometer readings. J. A. M. A., 1939, 113, 2381. 